\title{
127. Note on the Relations on Steenrod Algebra
}

\section{By Katuhiko Mizuno and Yoshihiro SaIto}

(Comm. by K. KunUGI, M.J.A., Nov. 12, 1959)

The object of this note is to show some relations of binomial coefficients $\bmod p$ where $p$ is a prime, and using of them to show some relations on the Steenrod algebra. We shall use the results of José Adem. ${ }^{1)}$

1. Relations of binomial coefficients. Let $A_{n}=\sum_{i=0}^{n}\left(\begin{array}{c}n-i \\ i\end{array}\right)$, where $n$ is any non-negative integer, so that

$$
A_{0}=\left(\begin{array}{l}
0 \\
0
\end{array}\right)=1, A_{1}=\left(\begin{array}{l}
1 \\
0
\end{array}\right)+\left(\begin{array}{l}
0 \\
1
\end{array}\right)=1, A_{2}=\left(\begin{array}{l}
2 \\
0
\end{array}\right)+\left(\begin{array}{l}
1 \\
1
\end{array}\right)+\left(\begin{array}{l}
0 \\
2
\end{array}\right)=2, \cdots \text {. }
$$

Generally

$$
\begin{aligned}
A_{n} & =\sum_{i=0}^{n}\left[\left(\begin{array}{c}
n-i-1 \\
i
\end{array}\right)+\left(\begin{array}{c}
n-i-1 \\
i-1
\end{array}\right)\right] \\
& =\sum_{i=0}^{n-1}\left(\begin{array}{c}
n-1-i \\
i
\end{array}\right)+\left(\begin{array}{c}
-1 \\
n
\end{array}\right)+\sum_{i=0}^{n-2}\left(\begin{array}{c}
n-2-i \\
i
\end{array}\right)+\left(\begin{array}{c}
n-1 \\
-1
\end{array}\right)+\left(\begin{array}{c}
-1 \\
n-1
\end{array}\right) \\
& =A_{n-1}+A_{n-2}+(-1)^{n}+(-1)^{n-1}=A_{n-1}+A_{n-2},
\end{aligned}
$$

then we have inductively

$$
A_{3 t} \equiv 1, A_{3 t+1} \equiv 1, A_{3 t+2} \equiv 0 \bmod 2 .
$$

Let $B_{b}^{\alpha}=\sum_{i=0}^{b}\left(\begin{array}{c}a+i(p-1) \\ b-i\end{array}\right)$ where $a$ is any number and $b$ is any non-negative integer, if $p=2$ it is easily recognized that $A_{n}=B_{n}^{0}$.

Then we will prove

$$
B_{b}^{\alpha}-B_{b-1}^{\alpha}+\cdots+(-1)^{i} B_{b-i}^{\alpha}+\cdots+(-1)^{p} B_{b-p}^{\alpha} \equiv\left(\begin{array}{l}
a \\
b
\end{array}\right) \bmod p .
$$

To prove this, deform $B_{b}^{a}$ in two ways;

and

$$
B_{b}^{\alpha}=\left(\begin{array}{l}
a \\
b
\end{array}\right)+B_{b-1}^{\alpha+(p-1)}
$$

$$
\begin{aligned}
B_{b}^{\alpha} & =\sum_{i=0}^{b}\left[\left(\begin{array}{c}
a-1+i(p-1) \\
b-i
\end{array}\right)+\left(\begin{array}{c}
a-1+i(p-1) \\
b-1-i
\end{array}\right)\right]=B_{b}^{\alpha-1}+B_{b-1}^{\alpha-1} \\
& =\left(\begin{array}{c}
p-1 \\
0
\end{array}\right) B_{b}^{\alpha-(p-1)}+\cdots+\left(\begin{array}{c}
p-1 \\
i
\end{array}\right) B_{b-i}^{\alpha-C(p-1)}+\cdots+\left(\begin{array}{c}
p-1 \\
p-1
\end{array}\right) B_{b-(p-1)}^{\alpha-(p-1)} \\
& \equiv B_{b}^{\alpha-(p-1)}+\cdots+(-1)^{i} B_{b-i}^{\alpha-(p-1)}+\cdots+(-1)^{p-1} B_{b-(p-1)}^{\alpha-(p-1)} \bmod p .
\end{aligned}
$$

Substituting the suitable expression (4) for the last term of (3) we have (2).

Hence from (4) and (2)

$$
B_{b+p}^{\alpha+(p-1)} \equiv(-1)^{p-1} B_{b}^{\alpha}+\left(\begin{array}{c}
a \\
b+p
\end{array}\right) \bmod p .
$$

Especially for any number $a$

1) José Adem: The Relations on Steenrod Powers of Cohomology Classes, Algebraic Geometry and Topology, Princeton University (1957). 
and if $0<b<p$

$$
B_{0}^{\alpha}=\left(\begin{array}{l}
a \\
0
\end{array}\right)=1
$$

$$
\begin{aligned}
B_{b}^{-1} & =\sum_{i=0}^{b}\left(\begin{array}{c}
-1+i(p-1) \\
b-i
\end{array}\right) \\
& =(-1)^{b}+\left(\begin{array}{c}
p-2 \\
b-1
\end{array}\right)+\left(\begin{array}{c}
p-3 \\
b-2
\end{array}\right)+\cdots+\left(\begin{array}{c}
p-(i+1) \\
b-i
\end{array}\right)+\cdots+\left(\begin{array}{c}
p-b-1 \\
0
\end{array}\right) \\
& =(-1)^{b}+\left(\begin{array}{c}
p-2 \\
p-b-1
\end{array}\right)+\left(\begin{array}{c}
p-3 \\
p-b-1
\end{array}\right)+\cdots+\left(\begin{array}{c}
p-(i+1) \\
p-b-1
\end{array}\right)+\cdots+\left(\begin{array}{c}
p-b-1 \\
p-b-1
\end{array}\right) \\
& =(-1)^{b}+\left(\begin{array}{l}
p-1 \\
p-b
\end{array}\right) \equiv(-1)^{b}+(-1)^{b-1}=0 \bmod p .
\end{aligned}
$$

2. Relations on Steenrod algebra. Let $p=2$. We shall calculate the number of $S q^{k-t} S q^{t}$ which appear in the admissible expansion of $\sum_{i=0}^{k} S q^{k-i} S q^{i}$ and we shall denote $S q^{i} S q^{j}$ simply by $(i, j)$ in the following discussion.

Let $k=3 n+m$ where $n$ is any non-negative integer and $m=0,1,2$. It is evident that if we expand the expression

$$
\begin{aligned}
(k, 0)+(k-1,1) & +(k-2,2)+\cdots+(k-t, t)+\cdots+(2 n+m, n) \\
& +(2 n+m-1, n+1)+\cdots+(2, k-2)+(1, k-1)+(0, k)
\end{aligned}
$$

as the admissible terms, $t$ is less than or equal to $n$ and $(k-t, t)$ appears from the back part of this expression except for the $(t+1)$-th term of the front part. We shall denote the number of $(k-t, t)$ in the admissible expansion of the above expression as $N_{m}^{n}(t)$ in the following.

By the Adem relation

$$
\begin{aligned}
N_{m}^{n}(t)=1 & +\left(\begin{array}{c}
n+1-t-1 \\
2 n+m-1-2 t
\end{array}\right)+\left(\begin{array}{c}
n+2-t-1 \\
2 n+m-2-2 t
\end{array}\right)+\cdots+\left(\begin{array}{c}
3 n+m-1-t-1 \\
1-2 t
\end{array}\right) \\
& +\left(\begin{array}{c}
3 n+m-t-1 \\
-2 t
\end{array}\right) \\
=1 & \left(\begin{array}{c}
0 \\
3(n-t)+m-1
\end{array}\right)+\cdots+\left(\begin{array}{c}
3(n-t)+m-1-(2 n-2 t+m) \\
2 n-2 t+m
\end{array}\right) \\
& +\left(\begin{array}{c}
3(n-t)+m-1-(2 n-2 t+m-1) \\
2 n-2 t+m-1
\end{array}\right)+\cdots+\left(\begin{array}{c}
3(n-t)+m-1 \\
0
\end{array}\right)
\end{aligned}
$$

because $2(n-t)+m+j>(n-t)-j-1=3(n-t)+m-1-(2 n-2 t+m+j)$ if $j$ is non-negative.

Hence

$$
N_{m}^{n}(t)=1+A_{3(n-t)+m-1} \text {. }
$$

Thus by the aid of (1)

$$
N_{m}^{n}(t) \equiv 0 \quad \bmod 2 \text { if } m=1,2 .
$$

It is evident from our definition that $N_{0}^{n}(n)=1$, then we have similarly

$$
N_{0}^{n}(t) \equiv 1 \bmod 2 \text { for any } t .
$$

From (8) and (9) we have

Proposition 1. $\sum_{i=0}^{k} S q^{k-i} S q^{i}=0$ if $k \neq 3 n$,

$$
\sum_{i=0}^{2 n-1} S q^{i} S q^{3 n-i}=0 \text {. }
$$


Since $2^{2 n} \equiv 1,2^{2 n-1} \equiv 2 \bmod 3$, we have

CoRollary 2. $\sum_{i=1}^{2^{j}-1} S q^{2 j-i} S q^{i}=0$ for any positive integer $j . \quad$ (12)

Let $p$ be an odd prime. We shall calculate the number of $S t_{p}^{k-t} S t_{p}^{t}$ which appear in the admissible expansion of $\sum_{i=0}^{i}(-1)^{i} S t_{p}^{i-i} S t_{p}^{i}$ if $k=(p+1) n+m$ where $n$ is any non-negative integer and $m=0,1,2$, $\cdots, p$. We shall use the same notations as in the case $p=2$.

By the Adem relation

$$
\begin{aligned}
N_{m}^{n}(t) & =(-1)^{t}\left[1+(-1)^{m} \sum_{i=1}^{p m+m}\left(\begin{array}{c}
(n+i-t)(p-1)-1 \\
p n+m-i-p t
\end{array}\right)\right] \\
& =(-1)^{t}\left[1+(-1)^{m} \sum_{i=1}^{(n-t) p+m}\left(\begin{array}{c}
(n-t)(p-1)-1+i(p-1) \\
(n-t) p+m-i
\end{array}\right)\right] .
\end{aligned}
$$

From our definition

$$
N_{0}^{n}(n)=(-1)^{n},
$$

and from (13) if $m=1,2, \cdots, p$ we have

$$
\begin{aligned}
N_{m}^{n}(n) & =(-1)^{n}\left[1+(-1)^{m}\left[\sum_{i=0}^{m}\left(\begin{array}{c}
-1+i(p-1) \\
m-i
\end{array}\right)-\left(\begin{array}{c}
-1 \\
m
\end{array}\right)\right]\right] \\
& =(-1)^{n}\left[1+(-1)^{m}\left[B_{m}^{-1}-(-1)^{m}\right]\right]=(-1)^{n+m} B_{m}^{-1} .
\end{aligned}
$$

By the aid of (7) we have

$$
N_{m}^{n}(n) \equiv 0 \bmod p \quad \text { for } m=1,2, \cdots, p-1 \text {, }
$$

and if $m=p$, using of (5) and (6), we have

$$
\begin{aligned}
N_{p}^{n}(n) & =(-1)^{n+p}\left[(-1)^{p-1} B_{0}^{-1-(p-1)}+\left(\begin{array}{c}
-p \\
p
\end{array}\right)\right] \\
& \equiv(-1)^{n+p}\left[(-1)^{p-1}+\left(\begin{array}{c}
-1 \\
1
\end{array}\right)\right]=0 \bmod p .
\end{aligned}
$$

If $n-t$ is positive we have from (13)

$$
\begin{aligned}
N_{m}^{n}(t) & =(-1)^{t}\left[1+(-1)^{m}\left[\sum_{i=0}^{(n-t) p+m}\left(\begin{array}{c}
(n-t)(p-1)-1+i(p-1) \\
(n-t) p+m-i
\end{array}\right)\right.\right. \\
& \left.\left.-\left(\begin{array}{c}
(n-t)(p-1)-1 \\
(n-t) p+m
\end{array}\right)\right]\right],
\end{aligned}
$$

since $(n-t) p+m>(n-t)(p-1)-1>0$, we have by the aid of (5)

$$
\begin{aligned}
& N_{m}^{n}(t)=(-1)^{t}\left[1+(-1)^{m} B_{(n-t) p+m}^{(n-t)(p-1)-1}\right] \\
& \equiv(-1)^{t}\left[1+(-1)^{m}\left[B_{(n-t-1) p+m}^{(n-t-1)(p-1)-1}+\left(\begin{array}{c}
(n-t-1)(p-1)-1 \\
(n-t) p+m
\end{array}\right)\right]\right] \bmod p . \\
& N_{m}^{n}(t) \equiv(-1)^{t}\left[1+(-1)^{m} B_{m}^{-1}+(-1)^{m}\left(\begin{array}{c}
-1 \\
p+m
\end{array}\right)\right] \\
& =(-1)^{t+m} B_{m}^{-1}=(-1)^{n-t} N_{m}^{n}(n) \bmod p \text {. }
\end{aligned}
$$

From (14), (15) and (16) we have

$N_{0}^{n}(t) \equiv(-1)^{t}$, and $N_{m}^{n}(t) \equiv 0 \quad$ if $m=1,2, \cdots, p \quad \bmod p$.

Combining the above results we have

Proposition 3. $\sum_{i=0}^{k}(-1)^{i} S t_{p}^{k-i} S t_{p}^{i}=0$ if $k \neq(p+1) n$,

$$
\sum_{i=0}^{p n-1}(-1)^{i} S t_{p}^{i} S t_{p}^{(p+1) n-i}=0 \text {. }
$$

Since $p^{2 n} \equiv 1, p^{2 n-1} \equiv-1 \bmod (p+1)$, we have 
Corollary 4.

$$
\sum_{i=0}^{p^{j}-1}(-1)^{i} S t_{p}^{p^{j}-i} S t_{p}^{i}=0 \text { for any positive integer } j .
$$

Let $c$ be the canonical anti-automorphism of the Steenrod algebra. ${ }^{2)}$ Then there is a relation:

Then we have

$$
c\left(S q^{k}\right)=\sum_{i=0}^{k-1} S q^{k-i} c\left(S q^{i}\right) .
$$

Therefore we have

$$
\begin{aligned}
\sum_{j=0}^{k-1} S q^{i} c\left(S q^{k-i}\right) & =\sum_{i=0}^{k-1} S q^{i}\left(\sum_{j=0}^{k-i-1} S q^{k-i-j} c\left(S q^{j}\right)\right) \\
& =\sum_{j=0}^{k-1}\left(\sum_{i=0}^{k-j-1} S q^{i} S q^{k-j-i}\right) c\left(S q^{j}\right) .
\end{aligned}
$$

$$
\begin{aligned}
\mathrm{S} q^{k}+c\left(S q^{k}\right) & =\sum_{j=0}^{k-1} S q^{k-j} c\left(S q^{j}\right)+\sum_{i=0}^{k-1} S q^{i} c\left(S q^{k-i}\right) \\
& =\sum_{j=0}^{k-1}\left(\sum_{i=0}^{k-j} S q^{i} S q^{k-j-i}\right) c\left(S q^{j}\right) .
\end{aligned}
$$

Now we take the notation $M_{2}(k)$ which is $\sum_{i=0}^{k} S q^{i} S q^{k-i}$.

Then we have

CoROLlaRY 5. $S q^{a}+c\left(S q^{a}\right)=\sum_{j=0}^{\alpha-1} M_{2}(\alpha-j) c\left(S q^{j}\right)$.

Let $p$ be an odd prime. We take the notation $M_{p}(k)$ which is $\sum_{i=0}^{i}(-1)^{i} S t_{p}^{i} S t_{p}^{k-i}$. By the similar way we have the following

CoROLlaRY 6. $(-1)^{a} S t_{p}^{a}-c\left(S t_{p}^{a}\right)=\sum_{j=0}^{a-1} M_{p}(a-j) c\left(S t_{p}^{j}\right)$.

3. On the 2-adic number. In this section we shall calculate some binomial coefficients $\bmod 2$, and by the aid of these results we show some relations on the Steenrod squares $S q^{i}(i=0,1, \cdots)$. We shall omit the sign " $\bmod 2 "$ in this section since there is no confusion.

Let $t$ be any non-negative integer if no restriction is set up, and let $r, h<k$ be any and every positive integers. We shall prove the following lemmas:

$$
\left(\begin{array}{c}
2^{h}+t \\
2 t
\end{array}\right) \equiv 1 \text { if and only if } t=2^{h}-2^{p} \text { or } 2^{h} \text { where } 0 \leqq p \leqq h .
$$

$$
\left(\begin{array}{c}
2^{k}+2^{h}+t \\
2 t+1
\end{array}\right) \equiv 1 \text { if and only if } t=2^{k}-2^{p}+2^{h}-1 \text { or } 2^{k}+2^{h}-1
$$

where $h<p \leqq k$. $\leqq k$.

$$
\left(\begin{array}{c}
2^{k}-2^{h}+t \\
2 t+1
\end{array}\right) \equiv 1 \text { if and only if } t=2^{k}-2^{p}+2^{h}-1 \text { where } h<p
$$

$$
\left(\begin{array}{c}
2^{h}+t-1 \\
2 t
\end{array}\right) \equiv 1 \text { if and only if } t=2^{h}-2^{p} \text { where } 0 \leqq p \leqq h .
$$

$$
\left(\begin{array}{c}
2^{k} r+2^{h}+t-1 \\
2 t
\end{array}\right) \equiv 1 \text { and } t \leqq 2^{h} \text { if and only if } t=2^{h}-2^{p} \text { or }
$$

$2^{h}$ where $0 \leqq p \leqq h$.

Proof of (7.2). Put $t=a_{0}+2 a_{1}+4 a_{2}+\cdots+2^{i} a_{i}+\cdots$ where $a_{i}=0$ or 1 , then it is obvious that $a_{k+1}=a_{k+2}=\cdots=0$ since $2^{k}+2^{h}+t$ is greater than or equal to $2 t+1$.

The 2-adic expansions of $2^{k}+2^{h}+t$ and $2 t+1$ are

$$
\begin{gathered}
2^{k}+2^{h}+t=a_{0}+2 a_{1}+\cdots+2^{h} b_{h}+2^{h+1} b_{h+1}+\cdots+2^{k} b_{k}+2^{k+1} b_{k+1}, \\
2 t+1=1+2 a_{0}+\cdots+2^{h} a_{h-1}+2^{h+1} a_{h}+\cdots+2^{k} a_{k-1}+2^{k+1} a_{k} .
\end{gathered}
$$

2) J. Milnor: The Steenrod algebra and its dual, Ann. Math., 67 (1958). 
Then our assumption is equivalent to the following inequalities

$$
\begin{gathered}
1 \leqq a_{0} \leqq a_{1} \leqq \cdots \leqq a_{h-1} \leqq b_{h}, a_{h} \leqq b_{h+1}, a_{h+1} \leqq b_{h+2}, \cdots, \\
a_{k-1} \leqq b_{k} \text { and } a_{k} \leqq b_{k+1},
\end{gathered}
$$

where $b_{h} \equiv a_{h}+1, b_{h+i}=a_{h+i}$ or $\equiv a_{h+i}+1$ for $0<i<k-h, b_{k} \equiv a_{k}+1$ or $b_{k}=a_{k}$ and only at the last case $b_{k+1}=1$.

Thus we have

$$
a_{0}=a_{1}=a_{2}=\cdots=a_{h-1}=b_{h}=1,
$$

namely $a_{h}=0$, and if $b_{h+1}=\cdots=b_{p-1}=0$ and $b_{p}=1$ then we have

$$
a_{h+1}=\cdots=a_{p-1}=0, a_{p}=a_{p+1}=\cdots=a_{k-1}=1, a_{k}=0
$$

where $h+1 \leqq p \leqq k-1$, and if $b_{h+1}=\cdots=b_{k}=0$ we have

$$
a_{h+1}=\cdots=a_{k-1}=0, a_{k}=1 \text {. }
$$

Hence we have the desirable result.

As the others can be proved in similar way, we omit the proofs.

In the following we shall denote $S q^{i} \cdots S q^{j}$ simply by $(i \cdots j)$.

From the above lemmas we have the following propositions:

(8.1) It appears the term $\left(2^{j}+2^{i}\right) j>i \geqq 0$ in the admissible expansion of $(s \cdot r) s, r>0$ if and only if $s=2^{p} i \leqq p<j$.

(8.2) It appears the term $\left(2^{j}-2^{i}\right) j>i>0$ in the admissible expansion of $(s \cdot r) s, r>0$ if and only if $s=2^{p}-2^{i} i<p<j$. sion

Proof of (8.1). At first we consider the case $i=0$. In the expres-

$$
(s \cdot r)=\sum_{t=0}^{\left[\frac{s}{2}\right]}\left(\begin{array}{c}
r-t-1 \\
s-2 t
\end{array}\right)(s+r-t \cdot t)
$$

we put $t=0$, then we may represent

$$
\left(\begin{array}{c}
r-1 \\
s
\end{array}\right)=\left(\begin{array}{c}
2^{j-1}+n \\
2^{j-1}-n
\end{array}\right)=\left(\begin{array}{c}
2^{j-1}+n \\
2 n
\end{array}\right)
$$

for some non-negative integer $n$.

Thus from the lemma (7.1), we obtain

since $s>0$.

$$
s=2^{j-1}-\left(2^{j-1}-2^{p}\right)=2^{p} \quad 0 \leqq p<j
$$

Next we consider the case $i>0$. In the above expression we may represent

$$
\left(\begin{array}{c}
r-1 \\
s
\end{array}\right)=\left(\begin{array}{c}
2^{j-1}+2^{i-1}+n \\
2^{j-1}+2^{i-1}-(n+1)
\end{array}\right)=\left(\begin{array}{c}
2^{j-1}+2^{i-1}+n \\
2 n+1
\end{array}\right)
$$

for some non-negative integer $n$.

Thus from the lemma (7.2), we obtain

since $s>0$.

$$
s=2^{j-1}+2^{i-1}-\left[\left(2^{j-1}-2^{p}+2^{i-1}-1\right)+1\right]=2^{p} \quad i \leqq p<j
$$

As the proof of (8.2) can be carried on similarly by the aid of the lemma (7.4) or (7.3), we omit it.

We shall calculate some types $(s \cdot r)$ using Lemma 7.

If $s=2 k$, we have

$$
(2 k \cdot r)=\sum_{i=0}^{k}\left(\begin{array}{c}
r-i-1 \\
2 k-2 i
\end{array}\right)(2 k+r-i \cdot i)=\sum_{t=0}^{k}\left(\begin{array}{c}
r-k+t-1 \\
2 t
\end{array}\right)(k+r+t \cdot k-t),
$$

and we use this formula in the following. 
If $j>0$, from (7.4) we have

$$
\left(2^{j} \cdot 2^{j}\right)=\sum_{p=0}^{j-1}\left(2^{j+1}-2^{p} \cdot 2^{p}\right) .
$$

If $i<j$, from (7.5) we have

$$
\left(2^{i} \cdot 2^{j}\right)=\left(2^{j}+2^{i}\right)+\sum_{p=0}^{i-1}\left(2^{j}+2^{i}-2^{p} \cdot 2^{p}\right) .
$$

If $j \geqq 2$, from (7.1) we have

$$
\left(2^{j-1} \cdot 2^{j-1}+1\right)=\left(2^{j}+1\right)+\sum_{p=0}^{j-2}\left(2^{j}+1-2^{p} \cdot 2^{p}\right) .
$$

If $j \geqq i+2$, from (7.5) we have

since $t \leqq 2^{i}$.

$$
\left(2^{i+1} \cdot 2^{j}-2^{i}\right)=\left(2^{i}+2^{j}\right)+\left(2^{j} \cdot 2^{i}\right)
$$

If $j \geqq i+2$, from (7.5) we have

$$
\left(2^{i} \cdot 2^{j}-2^{i+1}\right)=\left(2^{j}-2^{i}\right)+\sum_{p=0}^{i-1}\left(2^{j}-2^{i}-2^{p} \cdot 2^{p}\right) .
$$

As an application of these relations $(23), \cdots,(27)$ we shall show some relations each of which is convenient to calculation of the stable secondary cohomology operation.

From (25), we have

$$
\begin{aligned}
0= & \left(1 \cdot 2^{j}\right)+\left(2^{j-1} \cdot 2^{j-1}+1\right)+\sum_{p=0}^{j-2}\left(2^{j}+1-2^{p} \cdot 2^{p}\right) \\
= & \left(1 \cdot 2^{j}\right)+\left(2^{j-1}\right)\left[\left(2^{j-2} \cdot 2^{j-2}+1\right)+\sum_{p=0}^{j-3}\left(2^{j-1}+1-2^{p} \cdot 2^{p}\right)\right] \\
& \quad+\sum_{p=0}^{j-2}\left(2^{j}+1-2^{p} \cdot 2^{p}\right) .
\end{aligned}
$$

Thus we have

$$
\begin{aligned}
0=\left(1 \cdot 2^{j}\right) & +\left[\left(2^{j-1}+2^{j-2}+1\right)+\left(2^{j-1} \cdot 2^{j-2} \cdot 1\right)\right]\left(2^{j-2}\right) \\
& +\sum_{p=0}^{j-3}\left[\left(2^{j}+1-2^{p}\right)+\left(2^{j-1} \cdot 2^{j-1}+1-2^{p}\right)\right]\left(2^{p}\right) .
\end{aligned}
$$

If $j=i+2$, from $(24)$ and (26), we have

$$
0=\left(2^{i} \cdot 2^{i+2}\right)+\left(2^{i+2} \cdot 2^{i}\right)+\sum_{p=0}^{i-1}\left(2^{i+2}+2^{i}-2^{p} \cdot 2^{p}\right)+\left(2^{i+1} \cdot 2^{i+1}+2^{i}\right) .
$$

Thus from (24) we obtain

$$
\begin{aligned}
0=\left(2^{i} \cdot 2^{i+2}\right) & +\left(2^{i+1} \cdot 2^{i} \cdot 2^{i+1}\right)+\left(2^{i+2} \cdot 2^{i}\right) \\
& +\sum_{p=0}^{i-1}\left[\left(2^{i+2}+2^{i}-2^{p}\right)+\left(2^{i+1} \cdot 2^{i+1}+2^{i}-2^{p}\right)\right]\left(2^{p}\right) .
\end{aligned}
$$
have

If $j \geqq i+k+3, k \geqq 0$, by applying the relation (27) repeatedly, we

$$
\left(2^{j}-2^{i}\right)=\sum_{s=0}^{k} E_{s}^{k}\left(2^{i+s}\right)+\sum_{p=0}^{i-1} F_{p}^{k}\left(2^{p}\right)+\left(2^{i} \cdot 2^{i+1} \cdots 2^{i+k+1} \cdot 2^{j}-2^{i+k+2}\right)
$$
where $E_{s}^{k}=\left(2^{i} \cdot 2^{i+1} \cdots 2^{i+s}\right)\left[\left(2^{j}-2^{i+s+1}-2^{i+s}\right)+\left(2^{i+s+1}\right)\left[\left(2^{j}-2^{i+s+2}-2^{i+s}\right)\right.\right.$

$$
\left.+\cdots+\left(2^{i+k-1}\right)\left[\left(2^{j}-2^{i+k}-2^{i+s}\right)+\left(2^{i+k} \cdot 2^{j}-2^{i+k+1}-2^{i+s}\right)\right] \cdots\right] \text {, }
$$

and $F_{p}^{k}=\left[\left(2^{j}-2^{i}-2^{p}\right)+\left(2^{i}\right)\left[\left(2^{j}-2^{i+1}-2^{p}\right)+\left(2^{i+1}\right)\left[\left(2^{j}-2^{i+2}-2^{p}\right)+\cdots\right.\right.\right.$

$$
\left.\cdots+\left(2^{i+k-1}\right)\left[\left(2^{j}-2^{i+k}-2^{p}\right)+\left(2^{i+k} \cdot 2^{j}-2^{i+k+1}-2^{p}\right)\right] \cdots\right] \text {. }
$$

On the other hand, if $j \geqq i+3$ we have from (24) and (26)

$$
0=\left(2^{i} \cdot 2^{j}\right)+\left(2^{j} \cdot 2^{i}\right)+\sum_{p=0}^{i-1}\left(2^{j}+2^{i}-2^{p} \cdot 2^{p}\right)+\left(2^{i+1} \cdot 2^{j}-2^{i}\right)
$$

and if $j=i+k+3$, the last term of $(30)$ is

$$
\left(2^{i} \cdot 2^{i+1} \cdots 2^{i+k+1} \cdot 2^{i+k+2}\right)=\left(2^{i} \cdot 2^{i+1} \cdots 2^{j-2} \cdot 2^{j-1}\right) .
$$

Thus we have

$$
\begin{aligned}
0=\left(2^{i} \cdot 2^{j}\right) & +\left(2^{i+1} \cdot 2^{i} \cdot 2^{i+1} \cdots 2^{j-1}\right)+\left(2^{i+1} \cdot E_{j-i-3}^{j-i-3} \cdot 2^{j-3}\right)+\cdots \\
& +\left(2^{i+1} \cdot E_{1}^{j-i-3} \cdot 2^{i+1}\right)+\left[\left(2^{j}\right)+\left(2^{i+1} \cdot E_{0}^{j-i-3}\right)\right]\left(2^{i}\right) \\
& +\sum_{p=0}^{i-1}\left[\left(2^{j}+2^{i}-2^{p}\right)+\left(2^{i+1} \cdot F_{p}^{j-i-3}\right)\right]\left(2^{p}\right) .
\end{aligned}
$$

3) These relations are reported by J. Adem in the Proc. N.A.S. without the proof (1952).

4) Relation (28) is reported by N. Shimada on the Symposium of Topology at Toyama University (1959). 